Disponível em

http://www.anpad.org.br/rac

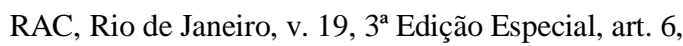
pp. 348-366, Outubro 2015

http://dx.doi.org/10.1590/1982-7849rac20152017

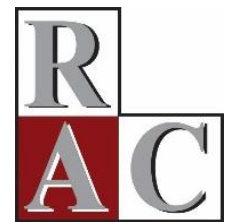

$(\mathrm{coc}) \mathrm{EY}$

\title{
Conexão Social Intraorganizacional, Suporte no Trabalho e Identificação Organizacional
}

\author{
Intra-organizational Social Connection, Support at Work and Organizational \\ Identification
}

Cristiano Oliveira Maciel

Pontifícia Universidade Católica do Paraná - PUCPR

Camila Camargo

Universidade Federal do Paraná - UFPR

Artigo recebido em 03.06.2014. Última versão recebida em 08.05.2015. Aprovado em 09.05.2015. Publicado online em 21.08.2015. 


\title{
Resumo
}

O objetivo do presente trabalho foi examinar em que medida os recursos relacionais, (a) suporte no trabalho (nas dimensões organização, chefe, colegas) e (b) conexão social intraorganizacional, estão associados à identificação organizacional. A realização de um survey com 209 participantes evidenciou que suporte do chefe não se relaciona significativamente à identificação organizacional, mas suporte da organização, suporte dos colegas e conexão social intraorganizacional, sim. Entretanto, verificou-se que a influência do suporte dos colegas é negativa sobre a identificação. Conclui-se que, em função da valência de seus efeitos, diferentes recursos relacionais podem tanto se complementar quanto concorrer pela variação no grau da identificação organizacional. Destaca-se, portanto, que as organizações devem ser compreendidas como constelações de laços sociais caracterizados por diferentes conteúdos, às vezes sinérgicos, outras vezes concorrentes, no que concerne à construção do sentido de unicidade do funcionário com sua organização.

Palavras-chave: conexão social intraorganizacional; suporte no trabalho; identificação organizacional.

\begin{abstract}
The aim of this study was to examine the extent to which relational resources, (a) support at work (in organizational, supervisor, and coworker dimensions) and (b) intra-organizational social connection, are associated with organizational identification. A survey with 209 participants showed that supervisor support was not significantly related to organizational identification, but organizational support, coworker support and intraorganizational social connection were. However, the influence of coworker support on identification was found to be negative. Thus, it was possible to conclude that, depending on the valence of their effects, different relational resources can be complementary or competitive in explaining variation in the degree of organizational identification. Therefore, organizations should be understood as constellations of social ties characterized by different contents, sometimes synergistic and others competing regarding construction of the uniqueness an employee has with an organization.
\end{abstract}

Key words: intra-organizational social connection; support at work; organizational identification. 


\section{Introdução}

A importância da identificação organizacional tem sido justificada pela sua influência em formas de pensar e comportamentos do indivíduo no trabalho (Sluss \& Ashforth, 2008). Vários estudos evidenciam que a identificação é uma preditora significativa de variáveis organizacionais importantes, como: aprendizagem, desempenho de tarefa, intenção de saída e produtividade (e.g., Fombelle, Jarvis, Ward, \& Ostrom, 2012; Hekman, Bigley, Steensma, \& Hereford, 2009; Millward \& Postmes, 2010; Mishra \& Bhatnagar, 2010; Walumbwa, Cropanzano, \& Hartnell, 2009). A extensa amplitude de elementos influenciados pela identificação organizacional decorre do fato de ela se caracterizar como uma ligação de natureza cognitiva e também afetiva. Ela revela em que medida alguém "se define por meio dos mesmos atributos que ele ou ela acredita definirem a organização" (Dutton, Dukerich, \& Harquail, 1994, p. 239). É possível até que essa ligação seja tão intensa a ponto de a identidade com a organização se tornar mais saliente que a identidade com outros grupos sociais. A principal consequência desse sentimento de unicidade entre indivíduo e organização é que aquilo que ocorre com a organização passa a ser percebido como consequência das realizações do próprio indivíduo (Mael \& Ashforth, 1992). Isso normalmente se dá em razão de o indivíduo julgar que sua organização de trabalho apresenta características que a distinguem, que a tornam atrativa e saliente (notável). Tal assertiva leva a concluir que atributos organizacionais assumidos como positivos são os principais elementos de explicação da força da identificação organizacional (Sluss \& Ashforth, 2008; Sluss, Ployhart, Cobb, \& Ashforth, 2012).

Entretanto, mais recentemente, vários estudos começaram a apontar que a identificação não decorre apenas de características da organização (Cooper \& Thatcher, 2010), mas de vários domínios (foci) relacionais (e.g., Jones \& Volpe, 2011; Knippenberg \& Schie, 2000; Schaubroeck, Peng, \& Hannah, 2013; Vuuren, Beelen, \& Jong, 2010; Wieseke, Kraus, Ahearne, \& Mikolon, 2012). O conteúdo dos laços sociais nas redes intraorganizacionais está quase que invariavelmente associado à identificação em função de as atividades de trabalho demandarem tanto recursos de tarefa (e.g., autonomia) quanto recursos de base relacional (e.g., confiança, suporte) para sua execução (Freeney \& Fellenz, 2013). Nesse sentido, a identificação é fruto não apenas de atributos organizacionais, como já ressaltado, mas também da arquitetura relacional do trabalho (Jones \& Volpe, 2011). Tal arquitetura vai além da estrutura imediata da atividade (i.e., job design) e contempla centralmente as interações e relacionamentos dos atores organizacionais (Grant, 2007).

Portanto, considerando os potenciais efeitos da arquitetura relacional do trabalho na percepção de unicidade entre indivíduo e organização (Grant, 2007), propõe-se aqui examinar a influência de dois recursos relacionais sobre a identificação organizacional: (a) suporte no trabalho (grau em que organização, chefe e colegas valorizam as contribuições do ator organizacional e se preocupam com seu bem-estar) (Hayton, Carnabuci, \& Eisenberger, 2012); e (b) conexão social intraorganizacional (percepção generalizada de que os contatos de interação mais frequente atuam no sentido de fazer o ator organizacional sentir-se incluído na rede intraorganizacional) (Maciel, 2014). Esses dois tipos de recursos foram escolhidos como antecedentes da identificação organizacional em razão de representarem diferentes parâmetros de avaliação de desempenho de papel dos atores sociais no trabalho. Isso significa que existem vários tipos de expectativas de comportamento de um funcionário em relação ao chefe imediato, em relação à organização e, sobretudo, em relação aos colegas de trabalho. A literatura sobre suporte no trabalho lembra que os indivíduos esperam que seus esforços sejam valorizados por colegas, chefe e organização. A noção de conexão social intraorganizacional chama atenção para a expectativa social de ser bem recebido pelos grupos sociais formalizados, especialmente pelos contatos de interação mais frequente no trabalho. Essa expectativa deriva da necessidade humana de afiliação a grupos sociais (Kadushin, 2012).

Apesar de já ser conhecida a influência da transposição da avaliação dos relacionamentos com os colegas ao sentido de unicidade entre indivíduo e organização (Sluss \& Ashforth, 2008), são muitos os caminhos ainda a percorrer no sentido de elencar quais, e com que efeitos, diferentes recursos relacionais, com origem em diferentes domínios, condicionam a força da identificação. Logo, a principal

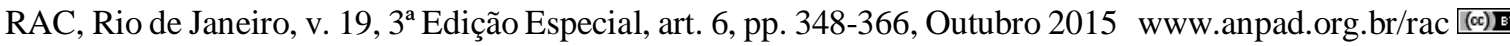


contribuição deste estudo é o apontamento de quais recursos relacionais ou que tipos de conteúdo de laços sociais com diferentes referentes (i.e., suporte da organização, do chefe, dos colegas, e conexão social intraorganizacional) se estendem ao grau de identificação organizacional.

Para consecução do objetivo de pesquisa, o presente artigo está estruturado em cinco seções. Após essa breve introdução, consta a revisão da literatura com o desenvolvimento das hipóteses a serem testadas. Na sequência, apresentam-se procedimentos metodológicos, resultados e conclusões.

\section{Revisão da Literatura e Hipóteses}

Conforme destacado, a atividade de trabalho demanda tanto recursos de tarefa quanto recursos relacionais (Freeney \& Fellenz, 2013). Logo, torna-se imprescindível a consideração da influência dos laços sociais na identificação organizacional. Nesse tipo de recurso, enquadram-se todos os elementos dentro do contexto social do trabalho que constituem o conteúdo das interações e relacionamentos dos atores organizacionais (Freeney \& Fellenz, 2013). Dutton, Dukerich e Harquail (1994) exemplificaram essa lógica ao afirmarem que o aumento da intensidade das interações diárias, em razão do tempo de participação na organização (tenure), faz com que as pessoas reconheçam umas às outras como membros cada vez mais naturalmente implicados no contexto organizacional. Walumbwa, Cropanzano e Hartnell (2009), bem como DeConinck (2011), reforçaram tal argumentação quando analisaram especificamente o relacionamento de membros organizacionais com seus líderes imediatos. Os dois estudos mostraram que a qualidade das interações sociais entre eles está significativamente correlacionada à identificação organizacional. Knight e Haslam (2010) verificaram que quando o controle gerencial burocrático alcança níveis que dificultam a interação social entre os indivíduos, há uma redução no grau de identificação organizacional. Davenport e Daellenbach (2011) enfatizaram o papel do capital social no processo de identificação em uma organização virtual. Os autores concluíram que mesmo em contextos caracterizados por pouca interação face a face, os relacionamentos intraorganizacionais condicionam significativamente a força da identificação organizacional.

Em resumo, tais estudos contribuem no sentido de mostrar de que forma ativos sociais de ordem relacional operam não apenas como insumos para manutenção e renovação da motivação para o trabalho, mas fundamentalmente como mecanismos de ligação do indivíduo com a sua organização (Grant, 2007). Sluss e Ashforth (2008) esclarecem que a associação entre relacionamentos no trabalho e identificação organizacional é estruturada por meio de mecanismos sociais que fazem com que a qualidade do relacionamento com a própria organização afete a identificação com ela (antropomorfizada), mas que os relacionamentos com chefes e colegas também são estendidos em alguma medida à organização. Esses mecanismos atuam no sentido de generalizar a atitude direcionada aos relacionamentos intraorganizacionais à atitude direcionada à organização. Nesse sentido, o suporte no trabalho se torna uma variável interessante porque considera diferentes domínios relacionais, como a valorização do trabalho pela organização, pelo chefe e pelos colegas. A conexão social intraorganizacional se diferencia do suporte dos colegas em função de se apresentar como outro tipo de conteúdo dos laços sociais (i.e., comportamento de inclusão) e por considerar tal conteúdo apenas por parte dos contatos de interação mais frequente do ator focal.

\section{Suporte no trabalho e identificação organizacional}

O conceito de suporte social no trabalho (ou suporte organizacional) foi desenvolvido por Eisenberger, Huntington, Hutchison e Sowa (1986) para evidenciar como indivíduos desenvolvem avaliações a respeito do quanto eles acham que sua organização de trabalho valoriza seus esforços e se preocupa com seu bem-estar. Os autores se apoiaram na perspectiva das trocas sociais e associaram o conceito a uma lógica de recursos de ordem relacional. A ênfase nos aspectos relacionais do suporte no trabalho contribuiu para destacar a necessidade de adicionar chefe e colegas também como fontes de suporte (e.g., Hayton et al., 2012). Essa apresentação tridimensional do construto (i.e., suporte da organização, do chefe e dos colegas) se mostrou interessante em função da ampliação das relações

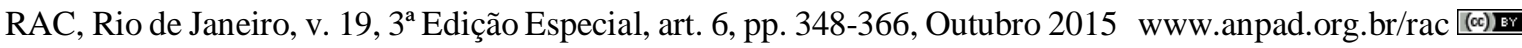


teóricas entre o conceito de suporte e seus antecedentes e consequentes. Em termos gerais, o conceito esclarece que a percepção da medida em que organização, chefe e colegas estão comprometidos com o funcionário tem reflexos no sentimento de ligação do indivíduo com a sua instituição de trabalho, bem como influencia outras atitudes e comportamentos positivos para as organizações (Rhoades \& Eisenberger, 2002). Eisenberger, Aselage, Sucharski e Jones (2004) esclarecem que a percepção de suporte gera um sentimento de obrigação (felt obligation) no funcionário e esse sentimento é condicionado pela influência das normas sociais de reciprocidade. Por sua vez, tais normas de reciprocidade (i.e., sentimento de dever com o outro) atuam fazendo com que o funcionário recompense a organização pelo tratamento positivo recebido por parte de chefe, colegas e organização.

A avaliação de que colegas se preocupam com os valores e sentimentos do indivíduo no trabalho, bem como de que chefe e organização constantemente consideram o seu bem-estar, contribui para criação de imagens de unicidade (Dutton et al., 1994). Assim, a intensidade de suporte social dado pela organização contribui diretamente para identificação com a própria organização. Já a intensidade do suporte de chefe e colegas é estendida ao nível organizacional por meio de generalizações. Como destacam Sluss e Ashforth (2008), a atitude desenvolvida em relação aos relacionamentos intraorganizacionais (e.g., chefe e colegas) é direcionada à atitude com relação à organização. Logo, afirma-se que o suporte da organização, do chefe e dos colegas influencia positivamente a força da identificação organizacional. Eisenberger et al. (1986) e Eisenberger et al. (2004) argumentam que a recompensa oferecida por esses três domínios (foci) vai ao encontro das necessidades básicas de aprovação e de orgulho, e esses sentimentos operam no sentido de fazer o indivíduo internalizar o status de papel de membro e o pertencimento à determinada organização em sua identidade (Frenkel \& Yu, 2011). Rhoades e Eisenberger (2002) afirmam ainda que a internalização do papel de membro dessa ou daquela organização decorre do fato de os recursos com origem nas diferentes fontes de suporte (i.e., organização, chefe, colegas) contribuírem para a manutenção de um sentimento de significado do próprio trabalho. Assim, tais argumentos sustentam as três hipóteses acerca das relações entre as dimensões do suporte no trabalho e a força da identificação organizacional:

$\mathbf{H}_{1}$ : O suporte da organização se relaciona positivamente à identificação organizacional.

$\mathbf{H}_{2}$ : O suporte do chefe se relaciona positivamente à identificação organizacional.

$\mathbf{H}_{3}$ : O suporte dos colegas se relaciona positivamente à identificação organizacional.

\section{Conexão social intraorganizacional e identificação organizacional}

A conexão social intraorganizacional revela a medida em que o ator focal (i.e., ego da rede) avalia que os contatos diretos (i.e., alters do ego) com os quais tem interação mais frequente no trabalho se comportam de modo a fazê-lo sentir-se incluído na organização. O conceito foi inspirado na noção de conexão social apresentada em estudos do campo da psicologia social (e.g., Park \& Maner, 2009; Seppala, Rossomando, \& Doty, 2013; Walton, Cohen, Cwir, \& Spencer, 2012) e na literatura da Análise de Redes Sociais (Maciel, 2014). Entretanto, a ideia de conexão social trabalhada na psicologia parece muito ampla para a análise organizacional em razão de contemplar vários tipos de relacionamentos, que são efetivamente diferenciados, como relacionamentos entre namorados, amigos e família. Por outro lado, a conexão social intraorganizacional se refere a um conteúdo específico das relações sociais (avaliação do papel dos contatos (alters) na inclusão do ego) limitado ao domínio das organizações de trabalho. Adicionalmente, a definição desse tipo de conexão dá conta da importância da influência dos contatos diretos com interações mais frequentes no comportamento (Evans \& Davis, 2005; Granovetter, 1973; Üstüner \& Iacobucci, 2012). Tal conceito vai ao encontro do crescente alerta de trabalhos que chamam atenção para a importância dos relacionamentos intraorganizacionais a fim de compreender melhor os antecedentes de emoções, atitudes, comportamentos e até de alterações fisiológicas do trabalhador (e.g., Dimotakis, Scott, \& Koopman, 2011; Gersick, Bartunek, \& Dutton, 2000; Heaphy \& Dutton, 2008; Liao, Liu, \& Loi, 2010; Ozcelik, 2013). Ademais, o exame do grau de conexão social intraorganizacional possibilita novas análises do comportamento do indivíduo no trabalho em função de ir além do exame estritamente estruturalista das redes sociais (e.g., Soltis, Agneessens, Sasovova, \& 
Labianca, 2013) e ainda considerar a primazia da necessidade básica do indivíduo por afiliação social (Friedkin, 2004; Kadushin, 2012).

No que concerne à sua influência na identificação, o grau de conexão social intraorganizacional atua como um mecanismo de qualificação das relações com os atores sociais de mais frequente interação no trabalho. As relações intraorganizacionais mais frequentes recebem então o status de relações familiares (Okhuysen, 2001). De acordo com Grant (2007), o aumento na frequência das interações contribui para aumentar mais o afeto positivo do que o afeto negativo entre os colegas de trabalho. Assim, se a avaliação global do grau de conexão intraorganizacional com os contatos no trabalho é alta, então os relacionamentos são qualificados como positivos. Se a conexão social intraorganizacional é alta, significa que os contatos (alters) se comportam fazendo o ego sentir: (a) que pertence à organização; (b) que está conectado à rede intraorganizacional; (c) que é parte de um grupo coeso; e (d) que faz parte de um grupo que pode ser considerado familiar. Logo, a avaliação positiva da qualidade dos relacionamentos com os contatos diretos e mais frequentes é estendida à qualidade do relacionamento com a organização e, por consequência, afeta a identificação (Sluss \& Ashforth, 2008). O estudo de Knippenberg e Schie (2000) exemplifica esse fenômeno. Os pesquisadores verificaram que a identificação com o grupo de trabalho (i.e., nível relacional) tem efeitos acentuados nas atitudes em relação à organização (i.e., nível organizacional). Entretanto, cabe ressaltar que as relações de grupo de trabalho, mesmo que apresentem sobreposições com os laços da conexão social intraorganizacional, são, quase sempre, diferentes das relações diretas e mais frequentes. Em resumo, tais argumentos permitem afirmar que um maior grau de conexão social intraorganizacional se estende, por meio da transferência de afeto e antropomorfização, à identificação organizacional (Sluss \& Ashforth, 2008).

$\mathbf{H}_{4}$ : A conexão social intraorganizacional se relaciona positivamente à identificação organizacional.

\section{Procedimentos Metodológicos}

O método selecionado para o estudo foi o survey interseccional. Esse método foi escolhido em função da possibilidade de uso de um questionário estruturado, o qual permitiria agregar mais facilmente, por meio das codificações, todas as respostas obtidas no levantamento. O survey interseccional é indicado quando se faz necessária a coleta de dados, num determinado momento, com a seleção de uma amostra para descrever características ou especificar relações entre variáveis (Babbie, 2005). Delimitou-se como contexto da pesquisa uma organização do setor de seguros, que não terá seu nome divulgado, conforme solicitação da própria empresa.

A organização foi escolhida em razão de ter um quadro composto majoritariamente por pessoas realizando atividades típicas de escritório, mas principalmente pelo acesso de um dos pesquisadores, que tinha um conhecido dentro da empresa. Esse contato já tinha feito mestrado e entendia, desta forma, a importância da pesquisa acadêmica. É importante ressaltar que nenhum dos autores da pesquisa faz ou já fez parte do quadro funcional da organização pesquisada. Atividades com essa natureza oferecem mais possibilidades de escolha dos contatos para interação e tempo para desenvolvimento dos laços, se comparado a organizações manufatureiras (Knight \& Haslam, 2010). A seguradora está instalada no sul do país e tem aproximadamente 700 funcionários.

A amostra foi não probabilística e por conveniência e adesão dos funcionários da empresa, portanto os resultados são passíveis de generalização apenas analítica (para a teoria), e não estatística. Nesse sentido é importante destacar: (a) as limitações impostas pela amostragem por adesão; e (b) as características contextuais que devem ser consideradas na leitura dos achados. Tayie (2005) aponta que as principais limitações da amostragem por adesão, além da falta de validade externa, centram no viés de autosseleção dos pesquisados, respondentes mais sensíveis a participar da pesquisa para demonstrar sua satisfação ou insatisfação, e na desconsideração de questões atitudinais e até genéticas relacionadas à prontidão a responder o questionário; os voluntários a responder pesquisas apresentam maior nível

RAC, Rio de Janeiro, v. 19, $3^{\text {a Edição Especial, art. 6, pp. 348-366, Outubro } 2015 \text { www.anpad.org.br/rac }(\propto c) \text { Er }}$ 
cultural, maior status ocupacional, maior necessidade de aprovação e são mais sociáveis. Ademais, a leitura dos resultados exige ter em mente que os dados foram coletados em apenas uma organização e, por isso, características particulares, que fogem da observação, interferem nas hipóteses testadas.

Com auxílio do departamento de RH da empresa, foram distribuídos questionários impressos a todos os funcionários e houve retorno de 255. Após exclusão de alguns com valores faltantes, foram analisados 209 questionários. A abordagem do pesquisador aos pesquisados consistiu em algumas etapas. Um representante do departamento de RH da empresa, sem cargo gerencial, conduziu um dos pesquisadores e o apresentou aos chefes dos departamentos da organização e pediu que o ajudassem em uma pesquisa de natureza acadêmica. Esse pesquisador foi apresentado pelos chefes de departamento como um professor que precisava fazer uma pesquisa. O pesquisador não ofereceu nenhum incentivo e esclareceu que não era preciso colocar o nome na pesquisa e que, assim que terminassem de responder o questionário, bastaria inseri-lo dobrado na caixa de papelão que tinha apenas uma abertura superior para depósito do mesmo. Todos foram avisados de que se tratava de uma pesquisa acadêmica, que não era um trabalho de consultoria e nem fazia parte de qualquer coleta de informações para a empresa. Ressaltou-se ainda que ninguém teria seu nome divulgado, e que, se não quisessem, poderiam não responder à pesquisa.

Uma limitação da análise dos questionários excluídos precisa ser ressaltada. É possível que características dos respondentes sejam responsáveis por sua decisão de não responder por completo o instrumento de coleta de dados. Seria necessário então comparar as características dos respondentes com aqueles que optaram por não responder ao questionário inteiro. Na segunda página, era solicitado que fossem respondidas questões quanto a características pessoais. Os valores faltantes se concentraram do final da primeira página até a segunda página, ou seja, exatamente as características pessoais é que não puderam ser levantadas, o que inviabilizou testes do impacto delas na quantidade de missing values.

O questionário estruturado foi composto por 6 indicadores sobre identificação (Mael \& Ashforth, 1992), 13 indicadores sobre suporte no trabalho (Hayton et al., 2012) e quatro indicadores de conexão social intraorganizacional (Maciel, 2014). Para que o respondente delimitasse seus contatos diretos nas suas respostas sobre conexão social, foi apresentada a explicação que consta no Apêndice. Os indicadores das escalas de identificação e suporte passaram pelo processo de tradução reversa. Os itens foram inicialmente traduzidos pelos autores deste estudo. Outro pesquisador, com fluência na língua inglesa, verteu os indicadores em português novamente para o inglês. As duas versões foram muito semelhantes. Empregou-se uma escala tipo Likert com 10 pontos, o que reduz a assimetria dos dados e contribui para aplicação de análises que pressupõem normalidade (Fornell, 1992). O questionário foi complementado com as variáveis de controle: sexo (0 masculino, 1 feminino), idade, estado civil (0 solteiro, 1 casado), grau de instrução ( 0 até médio, 1 superior), tempo de empresa e nível hierárquico (0 operacional, 1 acima).

\section{Validação das medidas}

Para avaliação da validade e confiabilidade das medidas, foi aplicada a análise fatorial confirmatória (AFC) (Hair, Anderson, Tatham, \& Black, 2009; Schumacker \& Lomax, 2004). O método de estimação empregado foi a máxima verossimilhança, pois nenhuma das variáveis intervalares apresentou assimetria fora dos limites de $\pm 1,50$, permitindo, portanto, aplicação de técnicas que pressuponham normalidade na distribuição dos dados (Schumacker \& Lomax, 2004). Todos os construtos foram tratados como indicadores reflexivos, como é mais comum nas mensurações psicométricas em estudos do comportamento organizacional. Os indicadores podem ser formativos ou reflexivos. Indicadores formativos não são necessariamente correlacionados, mas se assume que causam determinada variável latente, enquanto indicadores reflexivos pressupõem necessariamente correlação entre si e em conjunto não causam a variável latente, mas a revelam ou ajudam-na a emergir. Os indicadores reflexivos são apenas uma amostra das características da variável latente, enquanto indicadores formativos devem contemplar todas as causas da variável latente, por meio das variáveis observáveis (Kline, 2011). 
Os resultados mostram bons ajustes da matriz dos dados empíricos à matriz teórica. A matriz teórica é a matriz elaborada pelo pesquisador à luz da teoria. Nesse caso, é um modelo que relaciona as variáveis latentes da Análise Fatorial Confirmatória. A matriz dos dados empíricos é a matriz de dados gerados pela amostra da pesquisa. A comparação entre essas matrizes possibilita avaliar o quanto o padrão de covariância ou correlação dos dados empíricos se aproxima ou se distancia do padrão de relações especificadas pelo pesquisador. Nesse sentido, quando os ajustes do modelo são ruins é porque aquelas variáveis correlacionadas no modelo do pesquisador não aparecem de fato correlacionadas no plano empírico. O modelo apresentou o ajuste de $\chi^{2[504,06]} /$ Graus de Liberdade ${ }^{[220]}=2,29$, considerado bom frente aos valores recomendados entre 1 e 3 (Hair et al., 2009). Os indicadores RMSEA $(0,08)$, CFI $(0,93)$, IFI $(0,93)$, TLI $(0,92)$ também estão de acordo com as referências comumente sugeridas pela literatura (e.g., Schumacker \& Lomax, 2004).

A validade convergente dos indicadores em seus respectivos construtos foi verificada por meio da significância estatística ( $p$-value $<0,01$ ) das cargas padronizadas dos indicadores na AFC, conforme Tabela 1. A validade discriminante foi assegurada por meio do procedimento sugerido por Fornell e Larcker (1981). A correlação entre os fatores latentes foi elevada ao quadrado e então comparada à Variância Média Extraída (AVE). Como em nenhuma das díades a correlação foi superior à AVE, comprovou-se a validade discriminante dos construtos. Para análise da consistência interna dos fatores, foi calculado o Alfa de Cronbach (a). As análises de Variância Média Extraída (AVE) e de Confiabilidade Composta atestaram bons índices de confiabilidade dos construtos, conforme Tabela 1.

Tabela 1

\section{Análise Fatorial Confirmatória}

Descrição das Variáveis Manifestas e Confiabilidade das Variáveis Latentes

Carregamento

\section{Suporte Chefe (AVE=0,79; Confiabilidade Composta=0,95; $a=0,95$ )}

Meu chefe imediato realmente preocupa-se com o meu bem-estar.

$0,862 * * *$

Meu chefe imediato mostra que está pouco preocupado com o que acontece comigo. (I)

$0,799 * * *$

Meu chefe imediato realmente respeita meus objetivos e valores pessoais.

$0,952 * * *$

Meu chefe imediato está pronto para ajudar se eu precisar de um favor especial.

$0,871^{(*)}$

Meu chefe imediato fica orgulhoso se realizo bem o meu trabalho.

$0,950 * * *$

\section{Suporte Colegas $(\mathrm{AVE}=0,68 ;$ Confiabilidade Composta $=0,89 ; a=0,89)$}

Meus colegas de trabalho realmente respeitam meus objetivos e valores pessoais.

Meus colegas de trabalho ficam orgulhosos se realizo bem o meu trabalho.

Meus colegas de trabalho realmente preocupam-se com o meu bem-estar.

Meus colegas de trabalho valorizam se me preocupo com o bem-estar deles.

$0,843^{(*)}$

Suporte Organização (AVE=0,76; Confiabilidade Composta=0,92; $a=0,92$ )

Minha empresa está pronta para ajudar se eu precisar de um favor especial.

Minha empresa está pouco preocupada com o que acontece comigo. (I)

Minha empresa valoriza o modo como contribuo para o bem-estar dela.

$0,880 * * *$

Minha empresa realmente respeita meus objetivos e valores pessoais.

Identificação (AVE=0,67; Confiabilidade Composta=0,92; $a=0,91$ )

Quando alguém critica a empresa em que trabalho, eu me sinto pessoalmente insultado.

$0,719^{(*)}$

Eu fico muito curioso para saber o que os outros acham da empresa em que trabalho. 


\section{Tabela 1 (continuação)}

\begin{tabular}{lc}
\hline Descrição das Variáveis Manifestas e Confiabilidade das Variáveis Latentes & Carregamento \\
\hline Quando eu falo sobre esta empresa, eu normalmente digo "nós" ao invés de “eles". & $0,818^{* * *}$ \\
As conquistas dessa empresa em que trabalho são como minhas próprias conquistas. & $0,919 * * *$ \\
Quando alguém elogia a empresa em que trabalho, sinto como se eu fosse elogiado. & $0,929 * * *$ \\
Se uma notícia na mídia criticar esta empresa, provavelmente ficarei envergonhado. & $0,500^{* * *}$ \\
Conexão Social Intraorganizacional (AVE=0,71; Confiabilidade Composta=0,90; $\boldsymbol{a = 0 , 9 0 )}$ & $0,821^{(*)}$ \\
Meus contatos no trabalho fazem eu sentir que sou parte da organização. & $0,958^{* * *}$ \\
Meus contatos no trabalho fazem eu me sentir incluído na organização. & $0,875^{* * *}$ \\
Meus contatos no trabalho fazem eu sentir que sou parte de um grupo integrado. & $0,689 * * *$ \\
Meus contatos no trabalho fazem eu me sentir como se estivesse em casa. &
\end{tabular}

Nota. Fonte: elaborada pelos autores. (I): indicador invertido; $a$ : Alfa de Cronbach (Referência =0,60); AVE: Variância média extraída (Referência $=0,50)$.

*** $p<0,01,{ }^{(*)}$ : significância não calculada (parâmetro afixado em 1).

Mesmo tendo apresentado bons índices de ajustamento, o Modelo 1 ainda foi comparado a alternativas de estruturas fatoriais mais parcimoniosas, visando ressaltar a validade discriminante entre os construtos. No Modelo 2, os indicadores dos três tipos de suporte no trabalho foram combinados num modelo de segunda ordem. No Modelo 3, os indicadores de conexão e suporte dos colegas foram combinados num único fator. No Modelo 4 , todos os indicadores foram combinados num único fator. Verificou-se que existe diferença estatística entre o Modelo 1 comparado aos demais, mas todos produziram indicadores de ajustamento mais pobres, conforme Tabela 2.

Tabela 2

Comparação de Modelos da Análise Fatorial Confirmatória

\begin{tabular}{lccccccc}
\hline & $\chi^{2}$ & $g l$ & $\Delta \chi^{2}(g l)^{\text {a }}$ & RMSEA & CFI & IFI & TLI \\
\hline Modelo 1 & 504,06 & 220 & - & 0,08 & 0,93 & 0,93 & 0,92 \\
Modelo 2 & 1571,48 & 229 & $1067,42(09)^{* * *}$ & 0,17 & 0,67 & 0,67 & 0,64 \\
Modelo 3 & 1109,75 & 230 & $605,69(10)^{* * *}$ & 0,14 & 0,78 & 0,78 & 0,76 \\
Modelo 4 & 2769,83 & 230 & $2265,77(15)^{* * *}$ & 0,23 & 0,38 & 0,38 & 0,32 \\
\hline
\end{tabular}

Nota. Modelo 1 (estrutura de 5 fatores de primeira ordem). Modelo 2 (indicadores de suporte compondo um único fator de segunda ordem). Modelo 3 (indicadores de suporte dos colegas e conexão social intraorganizacional compondo um único fator de primeira ordem). Modelo 4 (todos os indicadores compondo um único fator de primeira ordem). Fonte: elaborada pelos autores.

${ }^{\text {a }}$ Teste relativo ao modelo 1 .

$* * * p<0,01$.

Uma última avaliação das escalas foi a análise do viés do método comum. Podsakoff, Mackenzie, Lee e Podsakoff (2003) esclarecem que o víes do método comum ocorre mais frequententemente quando é empregado um mesmo tipo de escala, com a mesma quantidade de opções de resposta e com corte transversal, ou seja, num único ponto no tempo. Os autores sugerem que seja verificado em que medida um estudo pode ser passível desse problema, pois a presença desse viés pode fazer com que a associação estatisticamente significativa entre duas variáveis ocorra simplesmente por causa do método comum (e.g., mesma escala e ponto no tempo) na coleta de dados.

A análise empregada aqui foi o teste do Fator Único de Harman. De acordo com Podsakoff et al. (2003), essa é a técnica mais amplamente empregada para avaliação do viés do método comum. Todos 
os indicadores foram submetidos a uma análise fatorial exploratória forçando a extração de um único fator. Quando a variância explicada da análise fatorial não ultrapassa 50\%, constata-se que o método comum empregado na coleta de dados não merece preocupação. No caso da presente pesquisa, a variância acumulada para os indicadores das escalas foi de 36,23, portanto, sem indicação de significativo víes do método comum.

\section{Análise e Discussão dos Resultados}

A análise dos dados teve início pelas estatísticas descritivas das variáveis de controle. Entre os respondentes, $58 \%$ são homens e $42 \%$ são mulheres, com 51,4\% deles casados. Mais de $88 \%$ dos participantes tem curso superior completo ou em andamento. 85\% ocupam cargos operacionais e 15\% cargo de supervisão ou nível acima (e.g., coordenação, gerência ou direção). A média de idade é de 31 anos. O tempo médio como funcionário na organização pesquisada é de aproximadamente 4 anos. Essas estatísticas descritivas, e outras como média e desvio-padrão, são importantes para compreensão mais acurada dos resultados do estudo. A observação das características de uma amostra não probabilística precisa ser levada em consideração na leitura das análises, pois em outras amostras não probabilísticas talvez os resultados não sejam os mesmos, por conta dessas diferenças no conjunto amostral.

Após análise das características da amostra por meio das variáveis de controle, as médias dos fatores latentes e as variáveis de razão (idade e tempo de empresa) tiveram a força e significância de suas associações verificadas por meio da correlação de Pearson, conforme resultados informados na Tabela 3. Para teste das hipóteses, foi empregada a análise de regressão múltipla, selecionando mínimos quadrados ordinários (OLS) como método de estimação. Foram desenvolvidos 6 modelos para exame da relação das variáveis de controle com a identificação e teste das hipóteses.

Tabela 3

Média, Desvio-padrão e Correlação entre Variáveis

\begin{tabular}{lccccccccc}
\hline & $\bar{x}$ & $\boldsymbol{S}$ & $\mathbf{1}$ & $\mathbf{2}$ & $\mathbf{3}$ & $\mathbf{4}$ & $\mathbf{5}$ & $\mathbf{6}$ & $\mathbf{7}$ \\
\hline 1. Idade & 31,00 & 10,63 & $1,00^{* * *}$ & & & & & & \\
2. Tempo de Empresa & 4,38 & 6,46 & $0,42^{* * * *}$ & $1,00^{* * *}$ & & & & & \\
3. Suporte da Organização & 6,98 & 1,67 & $-0,04$ & $-0,08$ & $1,00^{* * *}$ & & & & \\
4. Suporte do Chefe & 6,71 & 1,71 & $-0,05$ & $-0,14^{* *}$ & $0,48^{* * *}$ & $1,00^{* * *}$ & & & \\
5. Suporte dos Colegas & 6,21 & 1,35 & $-0,00$ & $0,12^{*}$ & $0,39^{* * *}$ & $0,37^{* * *}$ & $1,00^{* * *}$ & & \\
6. Conexão Social & 7,65 & 1,63 & $-0,06$ & $-0,04$ & $0,29 * * *$ & $0,43^{* * *}$ & $0,34^{* * *}$ & $1,00^{* * *}$ & \\
Intraorganizacional & & & & & & & & & \\
7. Identificação & 5,30 & 1,49 & 0,07 & $-0,01$ & $0,54^{* * *}$ & $0,31^{* * *}$ & 0,09 & $0,26^{* * *}$ & $1,00^{* * *}$ \\
Organizacional & & & & & & & & & \\
\hline
\end{tabular}

Nota. As variáveis Idade e Tempo de Empresa foram transformadas (Log) para análise das correlações. Fonte: elaborada pelos autores.

$* * * p<0,01$ (two-tailed); ** $p<0,05$ (two-tailed); * $p<0,10$ (two-tailed).

No Modelo, 1 foi testada a influência das variáveis de controle na identificação. No Modelo 2, as preditoras foram as variáveis de controle e o suporte da organização. No Modelo 3, foi adicionada a variável suporte do chefe, mantendo as variáveis independentes testadas anteriormente. No Modelo 4, foi seguido o mesmo procedimento, desta vez adicionando a variável suporte dos colegas. No Modelo 5 , apenas as variáveis de controle e a variável conexão social intraorganizacional foram inseridas. No Modelo 6, todas as variáveis foram inseridas ao mesmo tempo como variáveis preditoras da identificação organizacional. O teste do Modelo 1 apontou que apenas $5 \%\left(\mathrm{R}^{2}\right.$ Ajustado $=0,05, p$-value

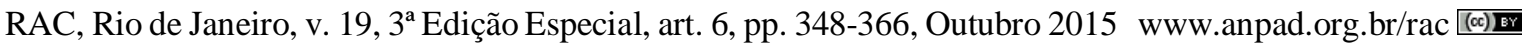


$<0,05)$ da variação da identificação se deve às variáveis de controle, mais precisamente ao cargo. Cargos acima do operacional se relacionam positivamente ao grau de identificação com a organização, principalmente em razão da responsabilidade e autonomia, como sugere a literatura. Essa associação é interessante porque reforça o argumento de que recursos de tarefa (e.g., autonomia encontrada em cargos acima do operacional) operam como recompensas ao indivíduo e, assim, contribuem à identificação (Eisenberger, Aselage, Sucharski, \& Jones, 2004). Cabe destacar ainda que a variável tempo de empresa, ao contrário do afirmado por Dutton et al. (1994), não se mostrou associada à identificação. Os Modelos 2, 3 e 4 mostram que a inserção das variáveis suporte da organização, suporte do chefe e dos colegas eleva o poder de explicação comparado às variáveis de controle, de um $\mathrm{R}^{2}$ Ajustado $=0,05$ para 0,33 . $\mathrm{O}$ Modelo 5 mostra que, quando considerada apenas a conexão social como variável de efeito principal, ela causa uma elevação do $R^{2}$ Ajustado $=0,05$ para 0,12 .

Para discussão dos resultados das hipóteses, foi considerado o Modelo 6, que contempla todas as variáveis de efeito principal e as de controle. Esse modelo apresentou $\mathrm{R}^{2}$ Ajustado $=0,35(\mathrm{~F}=11,37, p$ value $<0,01)$. A hipótese 1 , que relaciona o suporte da organização à identificação, foi comprovada $(\beta=0,55, p$-value $<0,01)$. Por outro lado, a relação entre suporte do chefe e a força da identificação, formalizada na hipótese 2 , não se mostrou estatisticamente significativa $(\beta=0,02, p$-value $>0,10)$. A hipótese 3, sobre a última dimensão do suporte no trabalho (i.e., suporte dos colegas) e sua relação com a identificação, também não foi comprovada, mas não em função do nível de significância, e sim da valência negativa do seu coeficiente padronizado na regressão $(\beta=-0,23$, $p$-value $<0,01)$. Isso quer dizer que existe de fato uma relação estatisticamente significativa entre essas variáveis, mas não como previsto na hipótese, pois à medida que o suporte dos colegas aumenta, a identificação com a organização é reduzida. Por sua vez, a hipótese 4 , que discorre sobre a relação entre o grau de conexão social intraorganizacional e a identificação com a organização, também foi comprovada $(\beta=0,17, p$ value $<0,05)$.

Por fim, mesmo tendo sido empregado um conjunto de variáveis de controle que poderia reduzir problemas de endogeneidade, recorreu-se também ao teste de Hausman para avaliar os potenciais efeitos de variáveis omitidas e de relações simultâneas entre variáveis independentes e a variável dependente (Wooldridge, 2010). Por exemplo, o suporte da organização e do chefe, que representa a organização, poderia ser afetado por um mesmo elemento. O teste de Hausman consiste em apurar se existem diferenças estatisticamente significativas entre as estimativas da regressão por Mínimos Quadrados Ordinários (MQO) e Mínimos Quadrados em Dois Estágios (MQ2E). As variáveis Suporte da Organização e Suporte do Chefe foram instrumentadas pela variável Conexão Social Intraorganizacional. Os resultados apontaram que as estimativas por Mínimos Quadrados Ordinários são consistentes, pois não existem diferenças estatisticamente significativas $(p$-value $<0,01)$. Os resultados de todos os modelos de regressão são apresentados na Tabela 4.

Tabela 4

\section{Resultados da Análise de Regressão OLS}

\begin{tabular}{lcccccc}
\hline & Modelo 1 & Modelo 2 & Modelo 3 & Modelo 4 & Modelo 5 & Modelo 6 \\
\hline Variáveis de Controle & & & & & & \\
Sexo & 0,03 & 0,05 & 0,06 & 0,07 & 0,04 & 0,07 \\
Idade (Log) & 0,03 & 0,02 & 0,02 & 0,00 & 0,06 & 0,02 \\
Estado Civil & 0,11 & $0,13^{*}$ & $0,13^{*}$ & $0,12^{*}$ & 0,08 & 0,10 \\
Nível de Instrução & $-0,03$ & 0,04 & 0,04 & 0,05 & $-0,04$ & 0,04 \\
Cargo & $0,24 * * *$ & 0,10 & 0,10 & $0,10^{*}$ & $0,23^{* * *}$ & $0,11^{*}$ \\
Tempo de Empresa (Logaritmo) & $-0,07$ & $-0,02$ & $-0,01$ & 0,03 & $-0,06$ & 0,03 \\
\hline
\end{tabular}


Tabela 4 (continuação)

\begin{tabular}{lcccccc}
\hline & Modelo 1 & Modelo 2 & Modelo 3 & Modelo 4 & Modelo 5 & Modelo 6 \\
\hline Variáveis de Efeito Principal & & & & & & \\
Suporte da Organização & & $0,53^{* * *}$ & $0,51^{* * *}$ & $0,57^{* * *}$ & & $0,55^{* * *}$ \\
Suporte do Chefe & & & 0,03 & 0,08 & & 0,02 \\
Suporte dos Colegas & & & & $-0,20^{* * *}$ & & $-0,23^{* * *}$ \\
Conexão Social Intraorganizacional & & & & & $0,27^{* * *}$ & $0,17^{* *}$ \\
\hline $\mathrm{F}$ & $2,67^{* * *}$ & $13,23^{* * * *}$ & $11,55^{* * *}$ & $11,67^{* * *}$ & $4,81^{* * *}$ & $11,37^{* * *}$ \\
$\mathrm{R}^{2}$ & 0,08 & 0,33 & 0,33 & 0,36 & 0,15 & 0,38 \\
$\mathrm{R}^{2}$ Ajustado & 0,05 & 0,30 & 0,30 & 0,33 & 0,12 & 0,35 \\
\hline
\end{tabular}

Nota. Fonte: elaborada pelos autores.

$* * * p<0,01$ (two-tailed); ** $p<0,05$ (two-tailed); * $p<0,10$ (two-tailed).

De modo geral, os resultados evidenciam que, em função da valência de seus efeitos, diferentes recursos relacionais podem tanto se complementar quanto concorrer pela variação no grau da identificação organizacional. Conforme destacado no Modelo 6 da análise de regressão, o suporte do chefe, fenômeno que ocorre numa importante esfera relacional das organizações (i.e., domínio subordinado-líder) (DeConinck, 2011; Walumbwa et al., 2009) não apresenta efeitos nem positivos nem negativos na identificação organizacional.

Tal achado enfraquece os argumentos que sustentaram a hipótese 2, com base em Sluss e Ashforth (2008) e Eisenberger et al. (2004), acerca de que a influência do suporte do chefe sobre a identificação organizacional derivaria de um sentimento de obrigação com o líder e que esse sentimento seria transferido à organização. A não comprovação dessa hipótese poderia, muito apressadamente, levar a crer que apenas a própria organização pode dar suporte social a ponto de afetar a identificação do funcionário com ela mesma. Sobretudo, tal conclusão pareceria plausível ao se observar que a hipótese 3 (suporte dos colegas influenciando a identificação) não foi comprovada e que a associação entre suporte organizacional e identificação, formalizada na hipótese 1 , é estatisticamente significativa.

Contudo, o peso das relações entre os colegas de trabalho, e principalmente entre os contatos de maior interação, deve ser mais cuidadosamente analisado ao se considerar as variáveis suporte dos colegas e conexão social intraorganizacional. De fato, o Modelo 3, que combina apenas as variáveis de controle com o suporte da organização, apresenta um bom poder de explicação $\left(\mathrm{R}^{2}\right.$ Ajustado $=0,30$ ), mas o Modelo 6, que inclui as variáveis de outros domínios relacionais (rede de colegas e rede de contatos diretos) eleva esse valor $\left(\mathrm{R}^{2}\right.$ Ajustado $\left.=0,35\right)$. Esse último modelo mostra que, mesmo não sendo comprovada a hipótese 2 , de que o suporte dos colegas influencia positivamente a identificação, há uma associação estatisticamente significativa entre as variáveis, entretanto, com valor negativo. Em adição, a comprovação da hipótese 4, que relaciona conexão social intraorganizacional à identificação, evidencia que ser incluído socialmente na organização pelos contatos diretos gera um sentimento de reciprocidade que é transferido em alguma medida à organização.

Logo, tais resultados levam a afirmar que, como ressaltado pela literatura (Eisenberger, Huntington, Hutchison, \& Sowa, 1986; Frenkel \& Yu, 2011), o peso das relações entre colegas e entre contatos de trabalho sobre a identificação do indivíduo com a organização não pode ser desconsiderado. Essencialmente, o suporte dos colegas deve ser compreendido como um recurso relacional que concorre com outros conteúdos dos laços sociais no trabalho no que concerne aos efeitos sobre a identificação. Tal conclusão, ao menos nesse ponto, é diametralmente oposta ao argumento de Sluss e Ashforth (2008), que consideram somente as sinergias dos laços sociais sobre a identificação. Nesse sentido, o papel competitivo do suporte dos colegas, em meio a outros recursos relacionais (i.e., suporte da organização, chefe e conexão social intraorganizacional), na variação da força da identificação organizacional, torna ainda maior a complexidade e a importância dos efeitos causados por um domínio relacional em outro, 
sobretudo quando comparado ao que fora previsto por Sluss e Ashforth (2008). Por sua vez, a ideia de conexão social intraorganizacional merece destaque ainda maior na tentativa de melhor compreender como os laços sociais no trabalho causam efeitos na identificação organizacional. Se o suporte dos chefes não é o caminho para maior identificação do funcionário com a organização e os colegas reduzem essa identificação à medida que parecem enfraquecer os efeitos da organização, resta à conexão social intraorganizacional a função de figurar como um importante elemento de nível meso na rede para estímulo ao sentimento de unicidade entre funcionário e organização. Em resumo, tais argumentos colocam em debate a possibilidade de fácil sinergia entre os diferentes domínios relacionais na identificação. Como visto, dependendo do conteúdo dos laços sociais, e da delimitação dos contatos do ator focal, os diferentes recursos relacionais podem ser concorrentes nos efeitos sobre a identificação.

\section{Conclusões}

A principal conclusão do presente trabalho é que as organizações devem ser compreendidas como constelações de laços sociais caracterizados por diferentes conteúdos. Alguns desses laços operam como recursos de estímulo à identificação com a organização e outros como limitadores desse processo. Portanto, recursos relacionais distintos podem se mostrar sinérgicos ou concorrentes no que concerne à construção do sentido de unicidade do funcionário com sua organização. Em adição, alguns conteúdos, como é o caso do suporte social na relação chefe-subordinado, podem não apresentar qualquer efeito quando se considera a identificação organizacional, mesmo que sejam comprovadamente importantes em outros aspectos do comportamento do indivíduo no trabalho.

Mais especificamente, como exemplificado pelos resultados já discutidos, laços de suporte das organizações com seus membros contribuem para aumento da identificação, conforme a primeira hipótese $\left(\mathrm{H}_{1}\right.$ : $\mathrm{O}$ suporte da organização se relaciona positivamente à identificação organizacional). A relação desse tipo de laço com a identificação amplia a discussão apresentada pela literatura clássica e mais sedimentada sobre identificação (e.g., Dutton et al., 1994; Mael \& Ashforth, 1992). Mael e Ashforth (1992), por exemplo, destacam o peso que se tem dado ao conjunto de características da própria organização. Normalmente, essas características notáveis são atributos de reputação, status e de legitimidade social, que diferenciam uma organização de outra. Corroborar a hipótese acerca da relação entre suporte da organização para com seus membros e a identificação destaca a importância das relações diádicas e sua influência no sentido de unicidade dos atores organizacionais com sua instituição de afiliação para desempenho do trabalho.

O suporte do chefe possivelmente tem efeitos sobre outras variáveis dependentes, mas não na identificação, diferentemente do que era esperado de acordo com a segunda hipótese $\left(\mathrm{H}_{2}\right.$ : $\mathrm{O}$ suporte do chefe se relaciona positivamente à identificação organizacional). A ausência de relação entre suporte do chefe e identificação é interessante em razão de destacar que, apesar de se reconhecer mais recentemente que os vários domínios relacionais impactam na identificação (Jones \& Volpe, 2011; Knippenberg \& Schie, 2000; Schaubroeck et al., 2013), nem todos esses domínios relacionais se comportam da mesma maneira, ou seja, contribuem invariavelmente de modo positivo para aumento da identificação organizacional.

O suporte dos colegas, por sua vez, reduz a força desse sentido de unicidade ao invés de contribuir positivamente com seu aumento, ou seja, não confirmou o esperado de acordo com a terceira hipótese $\left(\mathrm{H}_{3}\right.$ : $\mathrm{O}$ suporte dos colegas se relaciona positivamente à identificação organizacional). É importante especificar que o suporte dos colegas opera negativamente ao impedir a generalização da qualidade positiva das interações sociais do ator com seus pares à organização e ainda gera desidentificação (desidentification). Isso significa que o suporte social dos colegas centraliza seus efeitos positivos sobre seu próprio domínio e ainda gera efeitos negativos para a relação do ator social com sua organização no que se refere à identificação. Como já assinalado na discussão da hipótese 2, a relação entre suporte dos colegas e identificação, conforme hipótese 3 , reforça a ideia de que as relações sociais no interior das organizações merecem ser observadas com mais cuidado em função de sua complexidade. 
Diferentemente das generalizações propostas por Grant (2007), sobre laços sociais no trabalho e identificação, é preciso notar que cada tipo de laço apresenta um tipo de conteúdo, com valências, intensidades e efeitos distintos (Freeney \& Fellenz, 2013). Portanto, ao olhar para a estrutura dos tipos de suporte no trabalho, é preciso atentar ao fato de que talvez a literatura não esteja falando de um único construto (Rhoades \& Eisenberger, 2002), mas até talvez de construtos diferentes, a julgar pelos resultados do presente estudo.

E, quando o membro organizacional não se restringe a considerar somente aqueles que considera como colegas e leva em conta os alters (contatos diretos) com os quais mantém interação mais frequente, os efeitos positivos desse domínio (i.e., alters de contato mais frequente) são generalizados à organização. Quando a conexão social intraorganizacional é alta, isso faz com que a identificação organizacional aumente $\left(\mathrm{H}_{4}\right.$ : A conexão social intraorganizacional se relaciona positivamente à identificação organizacional). É importante ressaltar que, nesse conjunto de alters, podem ser considerados ao mesmo tempo indivíduos de diferentes domínios: domínio relacional dos colegas, do chefe e da organização antropomorfizada. $\mathrm{O}$ ator focal pode considerar pessoas do seu próprio grupo de trabalho ou de outros grupos, bem como podem ser contemplados colegas e chefe. Tais ponderações mostram que existem sobreposições e também diferenças de limites quando comparadas as fronteiras desses domínios relacionais, por exemplo: o conjunto de atores considerados na avaliação da conexão social intraorganizacional versus o conjunto de membros organizacionais incluídos na consideração do suporte social dos colegas nas organizações. Nesse sentido, a avaliação da conexão social intraorganizacional (Maciel, 2014) deve ser vista como uma atitude em relação a um domínio relacional com limites desenhados a partir da força dos laços no trabalho (Granovetter, 1973), enquanto os domínios relacionais de suporte dos colegas, chefe e organização são extraídos mais dedutivamente (Hayton et al., 2012; Rhoades \& Eisenberger, 2002), por meio de categorias com limites previamente estabelecidos por características mais formais, como a hierarquia, a cadeia de comando e o caráter institucional da organização.

Assim, ressalta-se que um comportamento específico relacionado à expectativa de papel dos seus contatos diretos, que é a percepção generalizada de que os contatos tentam incluir socialmente o ator focal no grupo organizacional, pode substituir os efeitos positivos que seriam esperados do suporte do chefe sobre a identificação, bem como atenuam os efeitos negativos do suporte dos colegas. Tais achados permitem evidenciar, além da força dos laços fracos, como feito por Granovetter (1973), também o potencial da força dos laços fortes na contribuição de resultados positivos para as organizações, como é o caso da identificação.

Por fim, a comprovação da relação positiva entre conexão social intraorganizacional e identificação organizacional e a comprovação dos efeitos negativos do suporte dos colegas torna ainda mais clara a complexidade dos efeitos dos diferentes domínios relacionais. Apesar de os dois tipos de recursos relacionais operarem essencialmente na esfera meso e mais provavelmente refletirem as interações horizontais na estrutura organizacional (Grant, 2007), o conteúdo e a demarcação distinta desses laços parecem evidenciar que são muitos os caminhos de influência da rede intraorganizacional sobre a identificação. Em adição, tais conclusões mostram que os argumentos de Sluss e Ashforth (2008) merecem ponderações, sobretudo no que concerne à competição de efeitos dos diferentes tipos de laços na variação do grau de identificação e quanto à reflexão sobre a delimitação das fronteiras dos diferentes domínios relacionais.

\section{Referências}

Babbie, E. (2005). Métodos de pesquisas de survey. Belo Horizonte: Editora UFMG.

Cooper, D., \& Thatcher, S. M. B. (2010). Identification in organizations: the role of self-concept orientations and identification motives. Academy of Management Review, 35(4), 516-538. doi: 10.5465/AMR.2010.53502693 
Davenport, S., \& Daellenbach, U. (2011). 'Belonging' to a virtual research centre: exploring the influence of social capital formation processes on member identification in a virtual organization. British Journal of Management, 22(1), 54-76. doi: 10.1111/j.1467-8551.2010.00713.x

DeConinck, J. B. (2011). The effects of ethical climate on organizational identification, supervisory trust, and turnover among salespeople. Journal of Business Research, 64(6), 617-624. doi: 10.1016/j.jbusres.2010.06.014

Dimotakis, N., Scott, B. A., \& Koopman, J. (2011). An experience sampling investigation of workplace interactions, affective states, and employee well-being. Journal of Organizational Behavior, 32(4), 572-588. doi: 10.1002/job.722

Dutton, J. E., Dukerich, J. M., \& Harquail, C. V. (1994). Organizational images and member identification. Administrative Science Quarterly, 39(2), 239-263. doi: 10.2307/2393235

Eisenberger, R., Aselage, J., Sucharski, I. L., \& Jones, J. R. (2004). Perceived organizational support. In J. Coyle-Shapiro, L. Shore, S. Taylor, \& L. Tetrick (Eds.), The employment relationship: examining psychological and contextual perspectives (pp. 206-225). Oxford: Oxford University Press.

Eisenberger, R., Huntington, R., Hutchison, S., \& Sowa, D. (1986). Perceived organizational support. Journal of Applied Psychology, 71(3), 500-507. doi: 10.1037/0021-9010.71.3.500

Evans, W. R., \& Davis, W. D. (2005). High-performance work systems and organizational performance: the mediating role of internal social structure. Journal of Management, 31(5), 758-775. doi: $10.1177 / 0149206305279370$

Fombelle, P. W., Jarvis, C. B., Ward, J., \& Ostrom, L. (2012). Leveraging customers` multiple identities: identity synergy as a driver of organizational identification. Journal of the Academy Marketing Science, 40(4), 587-604. doi: 10.1007/s11747-011-0254-5

Fornell, C. (1992). A national customer satisfaction barometer: the Swedish experience. Journal of Marketing, 56(1), 6-21. doi: 10.2307/1252129

Fornell, C., \& Larcker, D. F. (1981). Evaluating structural equation models with unobservable variables and measurement error. Journal of Marketing Research, 18(1), 39-50. doi: 10.2307/3151312

Freeney, Y., \& Fellenz, M. R. (2013). Work engagement, job design and the role of the social context at work: exploring antecedents from a relational perspective. Human Relations, 66(11), 14271445. doi: $10.1177 / 0018726713478245$

Frenkel, S. J., \& Yu, C. (2011). Managing coworker assistance through organizational identification. Human Performance, 24(5), 387-404. doi: 10.1080/08959285.2011.614301

Friedkin, N. E. (2004). Social cohesion. Annual Review of Sociology, 30, 409-425. doi: 10.1146/annurev.soc.30.012703.110625

Gersick, C. J. G., Bartunek, J. M., \& Dutton, J. E. (2000). Learning from academia: the importance of relationships in professional life. Academy of Management Journal, 43(6), 1026-1044. doi: $10.2307 / 1556333$

Granovetter, M. (1973). The strength of weak ties. American Journal of Sociology, 78(6), 1360-1380.

Grant, A. M. (2007). Relational job design and the motivation to make a prosocial difference. Academy of Management Review, 32(2), 393-417. doi: 10.2307/20159308

Hair, J. F., Jr., Anderson, R. E., Tatham, R. L., \& Black, W. C. (2009). Multivariate data analysis. New Jersey: Prentice-Hall, Inc. 
Hayton, J. C., Carnabuci, G., \& Eisenberger, R. (2012). With a little help from my colleagues: a social embeddedness approach to perceived organizational support. Journal of Organizational Behavior, 33(2), 235-249. doi: 10.1002/job.755

Heaphy, E. D., \& Dutton, J. E. (2008). Positive social interaction and the human body at work: linking organizations and physiology. Academy of Management Review, 33(1), 137-162. doi: $10.2307 / 20159380$

Hekman, D. R., Bigley, G. A., Steensma, H. K., \& Hereford, J. F. (2009). Combined effects of organizational and professional identification on the reciprocity dynamic for professional employees. Academy of Management Journal, 52(3), 506-526. doi: 10.5465/AMJ.2009.41330897

Jones, C., \& Volpe, E. H. (2011). Organizational identification: extending our understandings of social identities through social networks. Journal of Organizational Behavior, 32(3), 413-434. doi: $10.1002 /$ job.694

Kadushin, C. (2012). Understanding social networks: theories, concepts, and findings. New York: Oxford University Press.

Kline, R. B. (2011). Principles and practice of structural equation modeling. New York: Guilford Press.

Knight, C., \& Haslam, S. A. (2010). Your place or mine? Organizational identification and comfort as mediators of relationships between the managerial control of workspace and employees' satisfaction and well-being. British Journal of Management, 21(3), 717-735. doi: 10.1111/j.14678551.2009.00683.x

Knippenberg, D. van, \& Schie, E. C. M. van (2000). Foci and correlates of organizational identification. Journal of Occupational and Organizational Psychology, 73(2), 137-147. doi: $10.1348 / 096317900166949$

Liao, H., Liu, D., \& Loi, R. (2010). Looking at both sides of the social exchange coin: a social cognitive perspective on the joint effects of relationship quality and differentiation on creativity. Academy of Management Journal, 53(5), 1090-1109. doi: 10.5465/AMJ.2010.54533207

Maciel, C. O. (2014, novembro). Conexão social intraorganizacional: proposta conceitual e avaliação de seus efeitos em três domínios relacionais. Anais do Simpósio de Gestão da Inovação Tecnológica, Belo Horizonte, MG, Brasil, 28.

Mael, F., \& Ashforth, B. E. (1992). Alumni and their alma mater: a partial test of the reformulated model of organizational identification. Journal of Organizational Behavior, 13(2), 103-123. doi: $10.1002 /$ job. 4030130202

Millward, L. J., \& Postmes, T. (2010). Who we are affects how we do: the financial benefits of organizational identification. British Journal of Management, 21(2), 327-339. doi: 10.1111/j.1467-8551.2009.00667.x

Mishra, S. K., \& Bhatnagar, D. (2010). Linking emotional dissonance and organizational identification to turnover intention and emotional well-being: a study of medical representatives in India. Human Resource Management, 49(3), 401-419. doi: 10.1002/hrm.20362

Okhuysen, G. A. (2001). Structuring change: familiarity and formal interventions in problem-solving groups. Academy of Management Journal, 44(4), 794-808. doi: 10.2307/3069416

Ozcelik, H. (2013). An empirical analysis of surface acting in intra-organizational relationships. Journal of Organizational Behavior, 34(3), 291-309. doi: 10.1002/job.1798 
Park, L. E., \& Maner, J. K. (2009). Does self-threat promote social connection? The role of self-steem and contingencies of self-worth. Journal of Personality and Social Psychology, 96(1), 203-217. doi: $10.1037 / \mathrm{a} 0013933$

Podsakoff, P. M., MacKenzie, S. B., Lee, J.-Y., \& Podsakoff, N. P. (2003). Common method biases in behavioral research: a critical review of the literature and recommended remedies. Journal of Applied Psychology, 88(5), 879-903. doi: 10.1037/0021-9010.88.5.879

Rhoades, L., \& Eisenberger, R. (2002). Perceived organizational support: a review of the literature. Journal of Applied Psychology, 87(4), 698-714. doi: 10.1037/0021-9010.87.4.698

Schaubroeck, J. M., Peng, A. C., \& Hannah, S. T. (2013). Developing trust with peers and leaders: impacts on organizational identification and performance during entry. Academy of Management Journal, 56(4), 1148-1168. doi: 10.5465/amj.2011.0358

Schumacker, R. E., \& Lomax, R. G. (2004). A beginner's guide to structural equation modeling. Mahwah: Lawrence Erlbaum Associates Publishers.

Seppala, E., Rossomando, T., \& Doty, J. R. (2013). Social connection and compassion: important predictors of health and well-being. Social Research, 80(2), 411-430. doi: 10.1353/sor.2013.0027

Sluss, D. M., \& Ashforth, B. E. (2008). How relational and organizational identification converge: processes and conditions. Organization Science, 19(6), 807-823. doi: 10.1287/orsc.1070.0349

Sluss, D. M., Ployhart, R. E., Cobb, M. G., \& Ashforth, B. E. (2012). Generalizing newcomers` relational and organizational identifications: processes and prototypicality. Academy of Management Journal, 55(4), 949-975. doi: 10.5465/amj.2010.0420

Soltis, S. M., Agneessens, F., Sasovova, Z., \& Labianca G. (2013). A social network perspective on turnover intentions: the role of distributive justice and social support. Human Resource Management, 52(4), 561-584. doi: 10.1002/hrm.21542

Tayie, S. (2005). Research methods and writing research proposals. Cairu: CAPSCU.

Üstüner, T., \& Iacobucci, D. (2012). Does intraorganizational network embeddedness improve salespeople's effectiveness? A task contingency perspective. Journal of Personal Selling \& Sales Management, 32(2), 187-205. doi: 10.2753/PSS0885-3134320202

Vuuren, M. V., Beelen, P., \& Jong, M. D. T. (2010). Speaking of dominance, status diferences, and identification: making sense of a merger. Journal of Occupational and Organizational Psychology, 83(3), 627-643. doi: 10.1348/096317909X463661

Walton, G. M., Cohen, G. L., Cwir, D., \& Spencer, S. J. (2012). Mere belonging: the power of social connections. Journal of Personality and Social Psychology, 102(3), 513-532. doi: $10.1037 / \mathrm{a} 0025731$

Walumbwa, F. O., Cropanzano, R., \& Hartnell, C. A. (2009). Organizational justice, voluntary learning behavior, and job performance: a test of the mediating effects of identification and leader-member exchange. Journal of Organizational Behavior, 30(8), 1103-1126. doi: 10.1002/job.611

Wieseke, J., Kraus, F., Ahearne, M., \& Mikolon, S. (2012). Multiple identification foci and their countervailing effects on salespeople's negative headquarters stereotypes. Journal of Marketing, 76(1), 1-20. doi: 10.1509/jm.10.0444

Wooldridge, J. M. (2010). Introdução à econometria: uma abordagem moderna. São Paulo: Cengage Learning. 


\section{Dados dos Autores}

\section{Cristiano Oliveira Maciel}

Rua Imaculada Conceição, 1155, Bloco Acadêmico, Prado Velho, 80215-901, Curitiba, PR, Brasil. E-mail: cristiano.maciel@pucpr.br

Camila Camargo

Rua Prefeito Lothário Meissner, 632, Jardim Botânico, 80210-170, Curitiba, PR, Brasil. E-mail: caca.adm@ gmail.com 


\section{APÊNDICE}

\section{Orientações ao Pesquisado}

A Figura abaixo representa você e seus colegas (contatos diretos) no trabalho, as pessoas com as quais você interage com maior frequência. Pode ser amigos, chefes ou conhecidos com quem você passe mais tempo em interação. Não importa se são mais que 5 contatos ou menos, pois o desenho abaixo é apenas um exemplo. Pensando em você e esses colegas (contatos diretos), responda as afirmações a seguir:

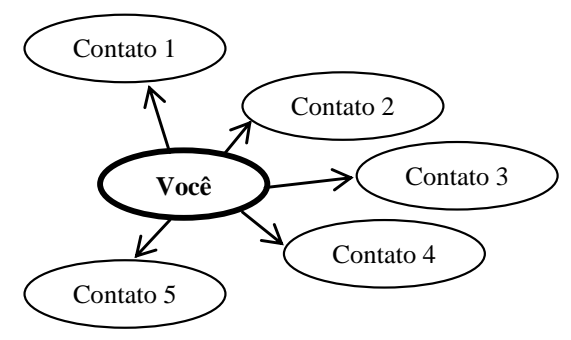

Figura 1. Orientações para Avaliação da Conexão Social Intraorganizacional 